\title{
Compact Liquid Crystal Based Tunable Band-Stop Filter with an Ultra-Wide Stopband by Using Wave Interference Technique
}

\author{
Longzhu Cai, Huan Xu, and Daping Chu \\ Electrical Engineering Division, University of Cambridge, Cambridge CB3 OFA, UK \\ Correspondence should be addressed to Daping Chu; dpc31@cam.ac.uk
}

Received 4 December 2016; Accepted 22 January 2017; Published 19 February 2017

Academic Editor: Luciano Tarricone

Copyright (C) 2017 Longzhu Cai et al. This is an open access article distributed under the Creative Commons Attribution License, which permits unrestricted use, distribution, and reproduction in any medium, provided the original work is properly cited.

\begin{abstract}
A wave interference filtering section that consists of three stubs of different lengths, each with an individual stopband of its own central frequency, is reported here for the design of band-stop filters (BSFs) with ultra-wide and sharp stopbands as well as large attenuation characteristics. The superposition of the individual stopbands provides the coverage over an ultra-wide frequency range. Equations and guidelines are presented for the application of a new wave interference technique to adjust the rejection level and width of its stopband. Based on that, an electrically tunable ultra-wide stopband BSF using a liquid crystal (LC) material for ultra-wideband (UWB) applications is designed. Careful treatment of the bent stubs, including impedance matching of the main microstrip line and bent stubs together with that of the SMA connectors and impedance adaptors, was carried out for the compactness and minimum insertion and reflection losses. The experimental results of the fabricated device agree very well with that of the simulation. The centre rejection frequency as measured can be tuned between 4.434 and $4.814 \mathrm{GHz}$ when a biased voltage of $0-20 \mathrm{Vrms}$ is used. The $3 \mathrm{~dB}$ and $25 \mathrm{~dB}$ stopband bandwidths were $4.86 \mathrm{GHz}$ and $2.51 \mathrm{GHz}$, respectively, which are larger than that of other recently reported LC based tunable BSFs.
\end{abstract}

\section{Introduction}

Band-stop filters (BSFs) with wide stopbands and compact structures are desirable in wireless communications because of their ability to suppress unwanted wideband noise and spurious signals [1]. In early 2002, the USA Federal Communications Commission (FCC) released the unlicensed frequency spectrum of ultra-wideband (UWB) $3.1-10.6 \mathrm{GHz}$ for commercial use, which has led to the further development of wide stopband BSFs [2]. According to the FCC's proposal, any bandwidth wider than $500 \mathrm{MHz}$ or $20 \%$ relative to the centre frequency is considered to be UWB [3]. Due to increasing levels of complexity and demands for advanced communication systems, UWB technology has drawn more and more attention $[4,5]$. The advantages of this technology consist of high resolution for ranging and geolocation and good resistance to small scale fading and narrowband interference $[3,6,7]$. Potential uses for UWB technology include medical imaging systems and vehicular radar systems $[3,6,7]$. Regulations regarding UWB standards vary across the world. For instance, in 2006, UWB transmission in Japan was allowed within the 3.1-4.8 and 6-10 GHz bands [3].

Some frequency bands, such as Worldwide interoperability for Microwave Access (WiMAX) with the frequency range 3.3-3.6 GHz and Wireless Local Area Network (WLAN) with the frequency spectra $5.15-5.35 \mathrm{GHz}$ and $5.725-5.825 \mathrm{GHz}$, are allocated within the designated UWB range, which can lead to interference when multiple UWB systems operate simultaneously within these bands [8]. To solve the issue of interference in the UWB frequency range, many types of BSFs have been proposed over the years, with various configurations [2, 9-12]. However, the reported BSFs usually have relatively complicated structures, large dimensions, and limited stopband bandwidths and attenuations. What is more, these filters are not reconfigurable. Liquid crystals (LCs) have been studied intensively as tunable dielectric materials in combination with microwave (MW) technology for many MW applications. This is owing to their ability for continuous tuning, large tuning range, relatively low dielectric loss in high frequencies, and cost effective fabrication process [13]. 


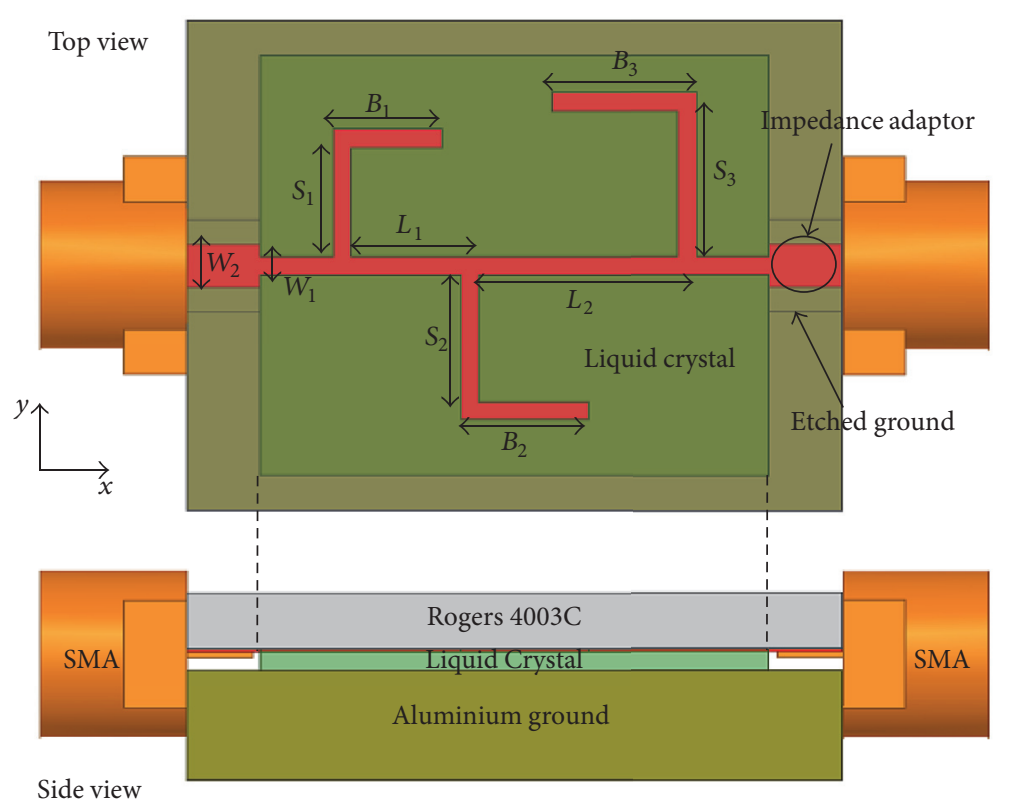

FIGURE 1: Schematic diagram of the proposed LC based tunable wideband BSF.

In this work, we demonstrate here an LC based tunable BSF with an inverted microstrip line (ML) structure and an ultra-wide and sharp stopband with large attenuation. The conventional and well-known open stub structures were widely used in filters to achieve frequency rejection behaviours $[14,15]$, with either open stubs of equal length or additional spurline structures between the stubs, for a wider rejection bandwidth and a high rejection ratio but with limited effects. In the past we presented an LC based tunable wideband BSF with double stubs of the same length as well [16]. Here our proposed new LC based tunable wideband BSF is based on a new substrate configuration and a modified bent stub structure with different stub lengths, which is more effective to attain a wider rejection bandwidth and a high rejection ratio without increasing the device's footprint. In this design we applied a new wave interference technique, instead of the traditional lumped equivalent circuit analysis, to optimize the filter performance with equations and guidelines for the adjustment of the rejection level and width of its stopband. SMA connectors are included in our simulation. Measured results from the fabricated device agree well with the simulated ones, and they exhibit a continually tuned rejection band with a centre frequency that can be adjusted between 4.434 and $4.814 \mathrm{GHz}$. The measured $3 \mathrm{~dB}$ and $25 \mathrm{~dB}$ bandwidths of the device are as high as $4.86 \mathrm{GHz}$ and $2.51 \mathrm{GHz}$, respectively. Furthermore, the attenuations in WiMAX and WLAN band ranges are both $<-25 \mathrm{~dB}$.

\section{Design of the Liquid Crystal Based Band-Stop Filter}

The configuration of the proposed LC based BSF is shown in Figure 1. A layer of LC material is sandwiched between an aluminium ground plate and $1.524 \mathrm{~mm}$ thick PCB-Rogers 4003C with relative permittivity $\varepsilon_{r}=3.55$ and dielectric loss $\tan \delta=0.0027$. The ML structure of the BSF is coated on the bottom of the Rogers $4003 \mathrm{C}$ to a thickness of $0.017 \mathrm{~mm}$. The thickness of the LC layer is $0.113 \mathrm{~mm}$, which is smaller than that of SMA connectors. To allow the SMA connectors to be inserted without touching the ground plate, two ends of the ground plate are shaped to leave a gap of $0.63 \mathrm{~mm}$. In this configuration, MW propagating into and out of the device experience a discontinued medium between air and the LC material, which causes impedance mismatching. To reduce the reflection, two impedance adaptors with width $W_{2}=$ $2.5 \mathrm{~mm}$ are designed at the two ends, as shown in Figure 1. The main ML and bent stubs have equal widths $W_{1}$, which are carefully designed to ensure that the characteristic impedance of the whole device, including the impedance adaptors and SMA connectors, thoroughly matches for minimising the device reflection and insertion loss $[17,18]$. Alignment layer made of polyimide mixture nylon with $0.2 \%$ concentration was coated on the Rogers and ground surfaces that are in contact with the LC material. After the curing process to evaporate the solvent in the polyimide mixture, the surfaces with the polyimide mixture are rubbed by a velvet cloth in order to align the LC molecules in a fixed direction.

The dimensions of the central ML structure shown in Figure 1 are as follows: $W_{1}=0.254 \mathrm{~mm}, S_{1}=B_{1}=3.85 \mathrm{~mm}$, $S_{2}=B_{2}=4.73 \mathrm{~mm}, S_{3}=B_{3}=6 \mathrm{~mm}, L_{1}=8.6 \mathrm{~mm}$, and $L_{2}=10.25 \mathrm{~mm}$. These dimensions have been adjusted and optimized by using a new wave interference technique to reject the WiMAX and WLAN bands at a high attenuation level during stopband tuning. In the following sections, the wave interference technique and device design procedure are presented in detail.

2.1. Liquid Crystal Used for the Band-Stop Filter. LC materials are known to be an optically and electrically anisotropic medium. The mechanism of nematic LC used as the tunable 


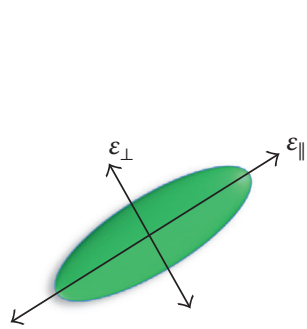

(a)

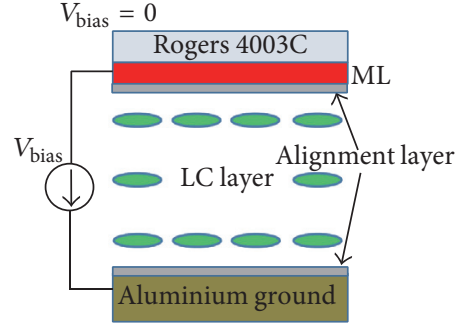

(b)

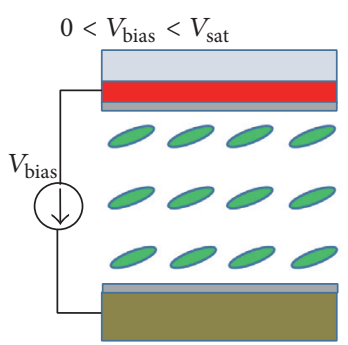

(c)

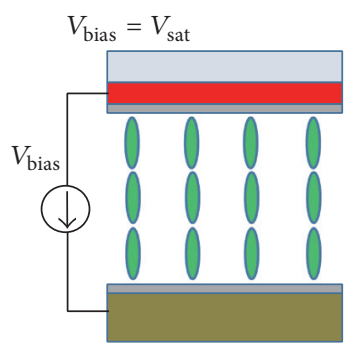

(d)

FiguRE 2: (a) Single LC molecule and states of LC molecules under various biased voltages. (b) $V_{\text {bias }}=0$, (c) $0<V_{\text {bias }}<V_{\text {sat }}$, and (d) $V_{\text {bias }}=V_{\text {sat }}$.

TABLE 1: Dielectric properties of the chosen LC material at 1-10 GHz and $23^{\circ} \mathrm{C}$.

\begin{tabular}{lllll}
\hline LC material & $\varepsilon_{\perp}$ & $\varepsilon_{\|}$ & $\tan \delta_{\perp}$ & $\tan \delta_{\|}$ \\
\hline GT3-24002 & 2.5 & 3.3 & 0.0123 & 0.0032 \\
\hline
\end{tabular}

dielectric material in MW devices is that the orientation of the rod-like nematic LC molecules can be reoriented by either an electric or a magnetic field, resulting in a change of the effective permittivity in the MW propagation path, which thus alters the device characteristic properties. Various molecular orientation states are illustrated in Figure 2, when LC molecules are subjected to different biased voltages.

The chosen nematic LC was GT3-24002 from Merck, synthesized with a wide nematic phase temperature range and with a clearing temperature of $\sim 173^{\circ} \mathrm{C}$, which makes this LC material suitable for many practical purposes [19]. The properties of GT3-24002 at $1-10 \mathrm{GHz}$ and $23^{\circ} \mathrm{C}$ are listed in Table 1.

\subsection{Wave Interference Techniques and Design of the Band-Stop}

Filter. The filter structure shown in Figure 1 comprises three bent stubs and two offset lines $\left(L_{1}\right.$ and $\left.L_{2}\right)$. In order to achieve a large attenuation as well as a sharp and wide stopband in a frequency range covering the WiMAX band and WLAN band, the physical length of each element must be carefully adjusted. A simple explanation of the complicated wave interference behaviour of the structure shown in Figure 1 starts from simpler structure with one stub, as shown in Figure 3(a). For this structure, a portion of MW is reflected from the stub to a traveling distance of $2 S_{1}$, at the intersection of the main transmission path and the stub. Destructive wave interference is expected if the MW fulfils the condition stated in (1). The stopband centre frequency for mode $n$ can be calculated according to (2) and (3):

$$
\begin{aligned}
\Delta S & =2 S_{1}=n \times \lambda_{g}+\frac{1}{2} \lambda_{g}, \quad(n=0,1,2, \ldots), \\
\lambda_{g} & =\frac{c}{f \times \sqrt{\varepsilon_{\mathrm{eff}}}}, \\
f & =\left(n+\frac{1}{2}\right) \frac{c}{2 S_{1} \times \sqrt{\varepsilon_{\mathrm{eff}}}},
\end{aligned}
$$

where $\lambda_{g}$ is the guided wavelength at the stopband centre frequency $f$ and $\varepsilon_{\text {eff }}$ is the effective relative permittivity when MW propagates throughout the structure. In (1), (1/2) $\lambda_{g}$ represents a $180^{\circ} \mathrm{MW}$ phase difference (antiphase). The length, $S_{1}=(1 / 4) \lambda_{g}$ for $n=0$, called the quarter-wavelength open stub, determines the required centre frequency of the fundamental stopband. Equation (3) indicates that stopband frequency $f$ is a function of both $S_{1}$ and $\varepsilon_{\text {eff }}$. For a fixed stub length $S_{1}, f$ can be adjusted by tuning the effective permittivity of the LC material using a biased voltage.

Figure $3(\mathrm{~b})$ is a conventional spurline structure, which has been used to realize LC based BSFs recently [20, 21]. With the same substrate and dimensions for the structures in Figures 3(a) and 3(b), the simulated transmission characteristics are shown in Figure 3(c), where we can see that the stub structure of Figure 3(a) has a wider stopband bandwidth and a larger attenuation at the rejection frequency. The simulation tool used in this work is High Frequency Structural Simulator (HFSS) from Ansys. The stronger coupling between the resonator of length $S_{1}$ and its parallel transmission line results in a narrower stopband bandwidth for the spurline structure. The larger attenuation for the stub structure comes from the same width of the stub and the main transmission line. Therefore using the prototype in Figure 3(a) is more advantageous to design wide stopband and large attenuation BSFs.

A new wave interference technique is used to explore the effects of the stub and offset lengths for BSFs that consist of several stubs and can also estimate the transmission properties of each structure by using this qualitative analysis. Only one stopband centre frequency can be achieved for the double-stub structure shown in Figure 4(a), assuming that the two stub lengths are equal $\left(S_{1}=S_{2}\right)$. The rejection level $\left|S_{21}\right|$ at the stopband centre frequency is governed by the offset transmission line length $L_{1}$, which is shown in Figure 4(b). By comparing the rejection levels for the onestub structure and the double-stub structure with offset length $L_{1}=0$, it can be shown that the double-stub structure can further reduce the rejection level by $\sim 5 \mathrm{~dB}$ due to the doubled destructive wave interference. However, a better attenuation at the centre frequency can be obtained if $L_{1}$ is around $(1 / 4) \lambda_{g}$ or $(3 / 4) \lambda_{g}$, as the consequence of another destructive wave interference occurring along the offset transmission line $L_{1}$. This is further confirmed by the low electric field distribution along the structure for $L_{1}=(1 / 4) \lambda_{g}$ 


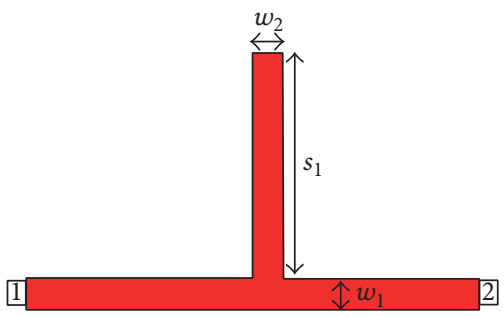

(a)

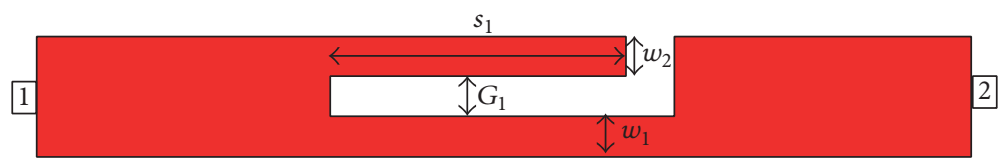

(b)

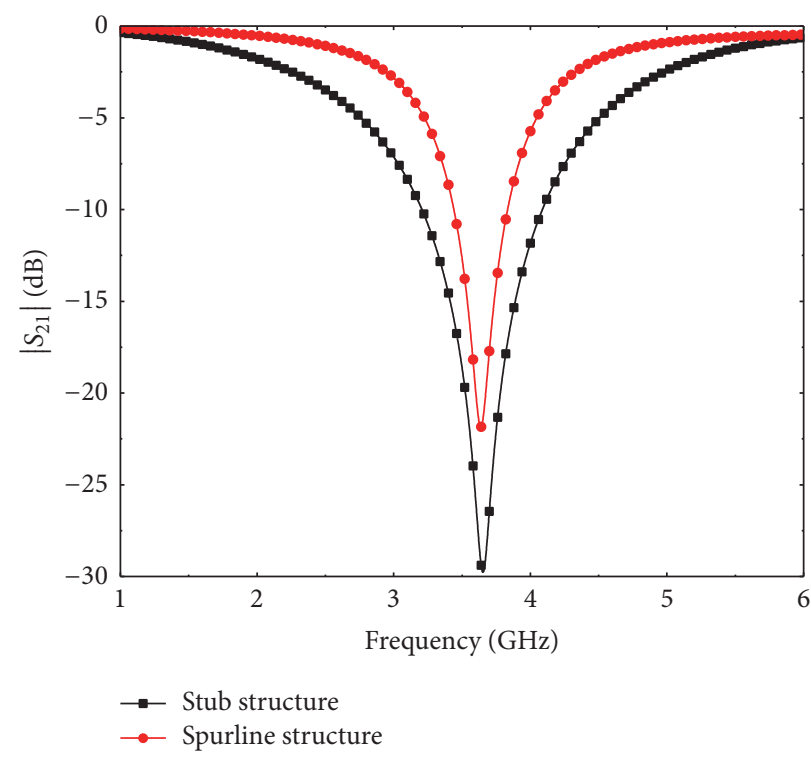

(c)

FIgURE 3: (a) One-stub structure, (b) spurline structure, and (c) their transmission properties for $w_{1}=w_{2}=0.276 \mathrm{~mm}$ and $G_{1}=0.05 \mathrm{~mm}$.

and $L_{1}=(3 / 4) \lambda_{g}$, as indicated in Figures $4(\mathrm{c})$ and $4(\mathrm{e})$, respectively.

Two valleys in a stopband are expected when $S_{1} \neq S_{2}$, each independently determined by the associated stub length that is described as $S_{1}=\lambda_{g 1} / 4$ and $S_{2}=\lambda_{g 2} / 4$. Figure 5(a) shows the transmission $\left|S_{21}\right|$ for the double-stub structure with different stub lengths, which clearly shows two valleys. In order to create a strong destructive wave interference between the two valleys, the offset length $L_{1}$ should be around $\lambda_{g 1} / 4$ and $\lambda_{g 2} / 4$. This is true if the two valleys are close enough, for example, as shown in Figure 5(b). The red line represents the insertion loss $\left|S_{21}\right|$, in such a condition $\left(S_{1}=\right.$ $9.5 \mathrm{~mm}, S_{2}=10.5 \mathrm{~mm}$, and $L_{1}=10.5 \mathrm{~mm}$ ), which achieves attenuation greater than $-40 \mathrm{~dB}$. However, the drawback of such a design is its narrow stopband width.

If the two stub lengths differ considerably, then the two valleys are separated by a few $\mathrm{GHz}$. As a result of wave interference of the individual stopbands, an undesirable overshoot appears between the two valleys that greatly reduces the attenuation. The double-stub structure is therefore not suitable for use as a BSF of large attenuation and wide stopband. To solve the problem, a third stub with a different length is added to the structure, which is expected to create a main stopband located between the two valleys that provides a strong attenuation as well as a sharp and wide stopband.

In order to minimise the interference from the popularly used WiMAX and WLAN bands in UWB applications, the lengths of two stubs can be roughly determined to produce transmission valleys at a minimum and a maximum frequencies, with the third stub of a length between that of these two stubs placed in the middle to produce a main stopband between the two valleys. Subsequently, based on the analysis of various offset length effects, the distances between these stubs are optimized to avoid undesirable overshoots. The three stubs are bent for compactness, and all the dimensions of the filter are carefully optimized and adjusted in the HFSS in order to obtain good characteristics, such as minimum insertion and reflection losses, a strong attenuation, and a sharp and wide stopband. The proposed bent three-stub structure was shown in Figure 1, and the final dimensions as optimized are reported in Section 2. Figure 6 shows the simulated scattering parameters ( $S$-parameters) $\left|S_{21}\right|$ and $\left|S_{11}\right|$ of the proposed BSF with SMA connectors included. To illustrate the properties of device transmission, Figure 7 shows the MW electric field distributions (including the SMA connection interface) at the untuned LC state (biased 


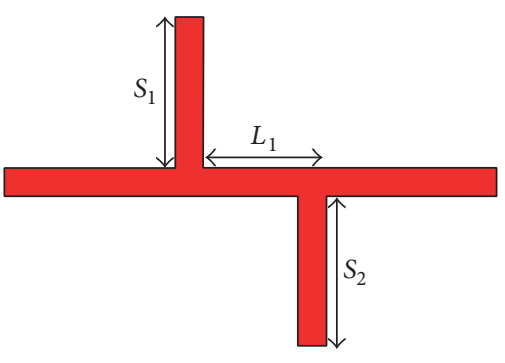

(a)

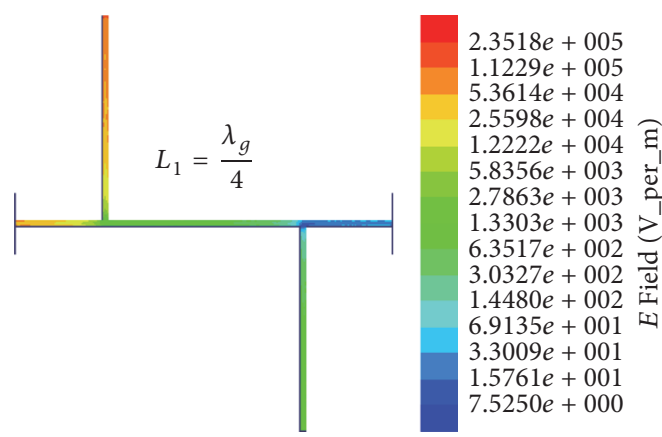

(c)

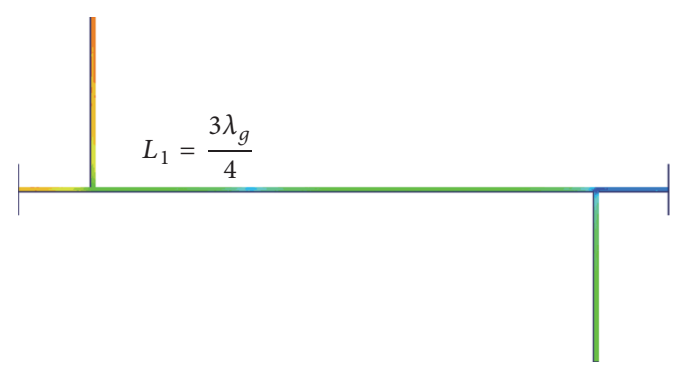

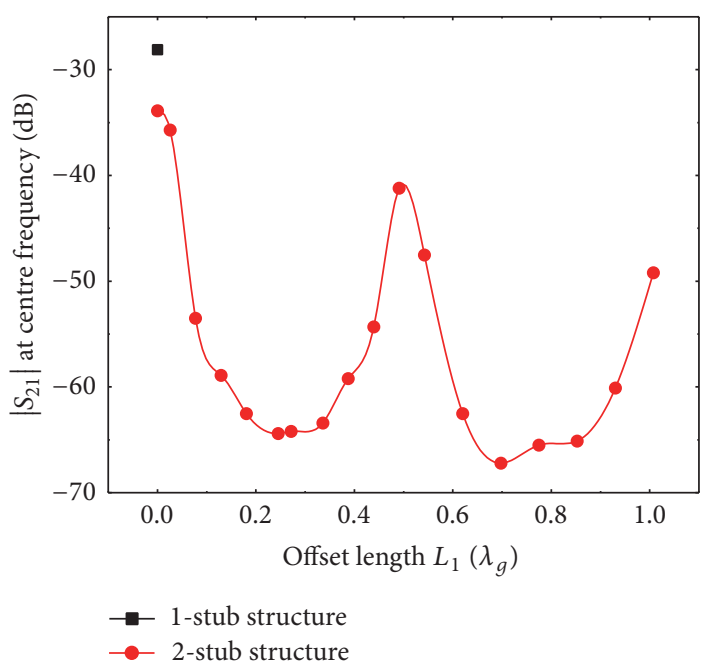

(b)

(e)

Figure 4: (a) Double-stub structure and (b) its transmission $\left(\left|S_{21}\right|\right)$ for $S_{1}=S_{2}$ at the centre frequency with respect to offset length $L_{1}$, and the electric field distribution when the offset lengths $L_{1}$ are (c) $(1 / 4) \lambda_{g}$, (d) $(1 / 2) \lambda_{g}$, and (e) $(3 / 4) \lambda_{g}$.

voltage $=0$ Vrms $)$ for two passbands $(2 \mathrm{GHz}$ and $8 \mathrm{GHz})$ and one stopband $(4.757 \mathrm{GHz})$. The color difference associated with the electric field strength clearly demonstrates an effective performance of the proposed BSF in rejecting the intended frequencies.

Figure 6 shows that the transmission $\left|S_{21}\right|$ has quite a sharp slope. The $3 \mathrm{~dB}$ and $25 \mathrm{~dB}$ bandwidths are as high as $4.73 \mathrm{GHz}$ and $2.54 \mathrm{GHz}$, respectively, in the untuned LC state. The fractional bandwidth (FBW) is defined as [23]

$$
\text { FBW }=\frac{\text { bandwidth }}{\text { centre frequency }}=\frac{f_{2}-f_{1}}{\left(f_{2}+f_{1}\right) / 2},
$$

where $f_{2}$ and $f_{1}$ are the upper and lower frequencies in the stopband, respectively. Hence the $3 \mathrm{~dB}$ and $25 \mathrm{~dB}$ FBW are $96.8 \%$ and $52.6 \%$, respectively. In addition, the stopband centre frequency can be continuously tuned between 4.337 and $4.757 \mathrm{GHz}$ with $9.7 \%$ tunability, relative to the centre frequency in the fully biased state. Moreover, during the tuning process the rejection level of transmission $\left|S_{21}\right|$ remains below $-50 \mathrm{~dB}$. Using such a BSF, frequency bands where wave interference may occur in UWB communications, such as the WiMAX and WLAN bands, will be effectively rejected to a level lower than $-30 \mathrm{~dB}$. This makes the proposed BSF particularly useful for UWB applications. 


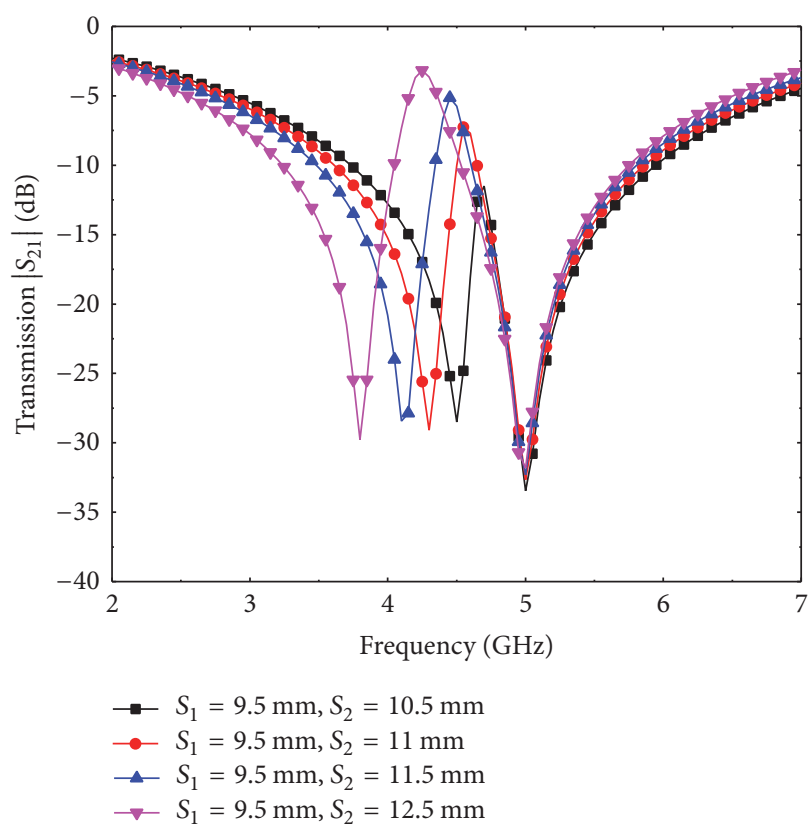

(a)

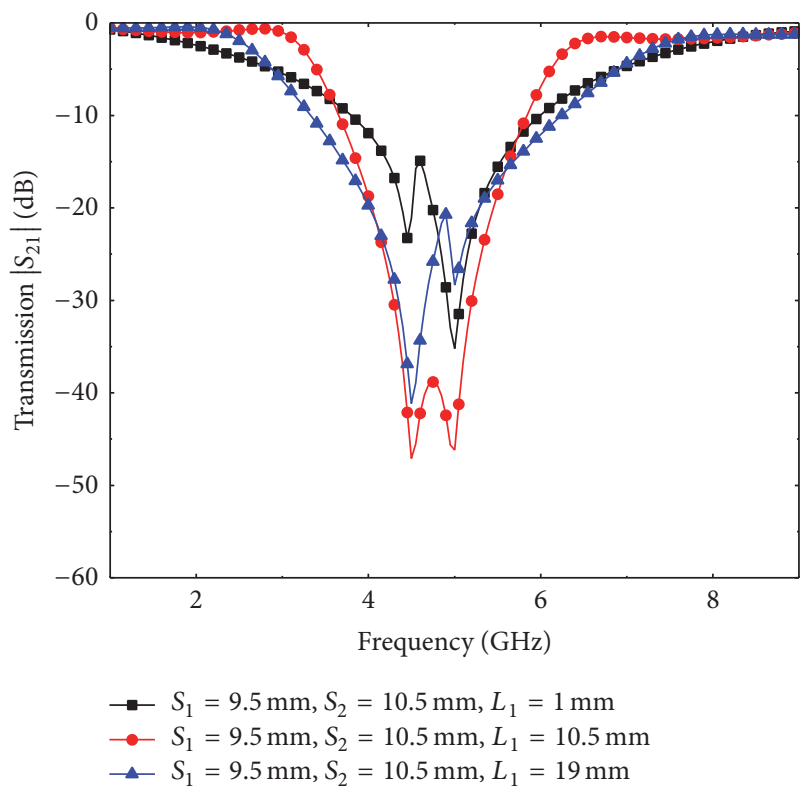

(b)

FIGURE 5: Transmission $\left|S_{21}\right|$ properties of the double-stub structure with (a) various stub length $S_{2}$ and (b) various offset length $L_{1}$.

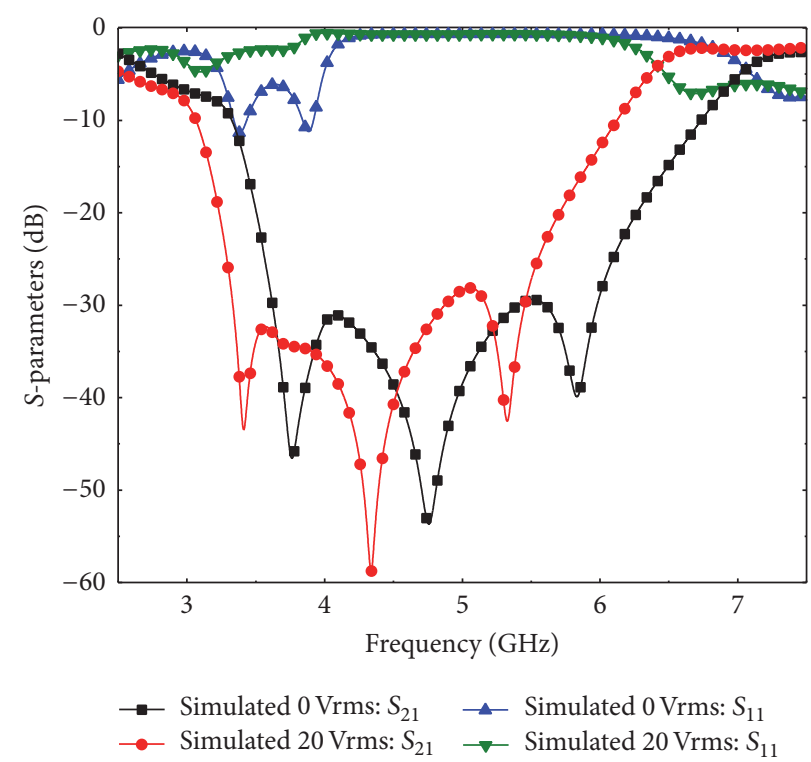

FIgURE 6: Simulated $S$-parameters of the proposed filter when the substrate LC is tuned.

\section{Experimental Results and Discussions}

The designed BSF was fabricated at a compact size of $34 \mathrm{~mm}$ $(L)$ by $22 \mathrm{~mm}(W)$, weighing $23.11 \mathrm{~g}$. These parameters are shown in Figure 8.

The device was measured using a two-port vector network analyser (VNA) Agilent 8722ET. The measured transmission $\left|S_{21}\right|$ under various driven voltages is displayed in Figure 9(a). At zero biased voltage the centre rejection frequency of the fabricated BSF is $4.814 \mathrm{GHz}$, and the LC layer exhibits the lowest dielectric permittivity $\varepsilon_{\perp}$. As the biased voltage increases, the LC layer permittivity increases as well, which results in a decrease of the device rejection frequency. The lowest rejection frequency reaches $4.434 \mathrm{GHz}$ when a biased voltage of $20 \mathrm{Vrms}$ is used, and the LC layer permittivity has a value of around $\varepsilon_{\|}$. The total tuned frequency range is $0.38 \mathrm{GHz}$, corresponding to a tunability of $8.6 \%$ relative to the centre frequency at a biased voltage of $20 \mathrm{Vrms}$. This is fairly close to 

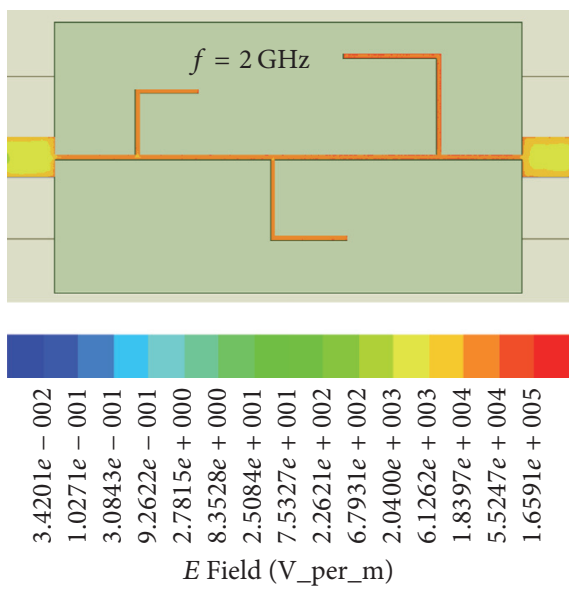

(a)
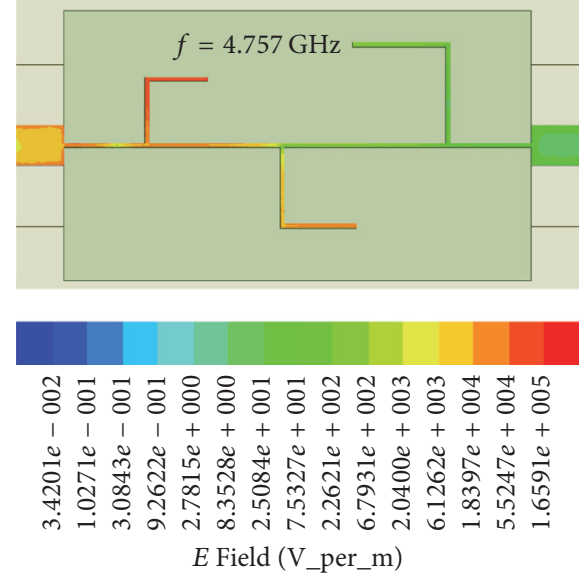

(b)
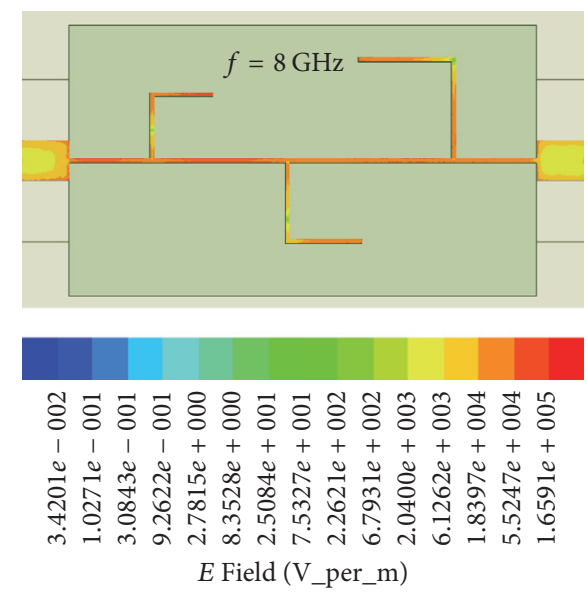

(c)

FIGURE 7: Electric field distributions of the proposed filter in the untuned state at its (a) passband $2 \mathrm{GHz}$, (b) stopband $4.757 \mathrm{GHz}$, and (c) passband $8 \mathrm{GHz}$.

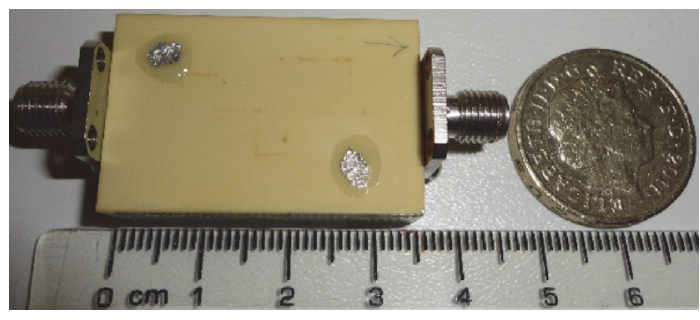

(a)

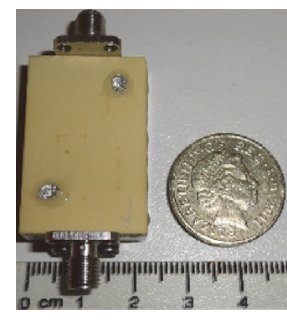

(b)

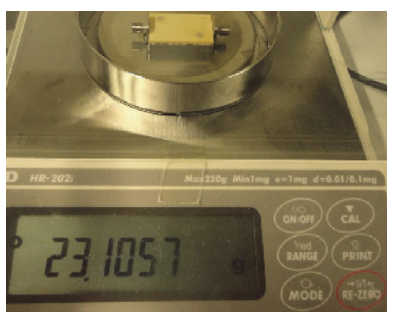

(c)

FIGURE 8: (a) The length, (b) width, and (c) weight of the fabricated BSF.

the targeted value of $9.7 \%$ tunability identified in simulation. The tuned frequencies with respect to the applied voltage are shown in Figure 9(b). The voltage range for the tuned frequency $8 \%-85 \%$ is $\sim 6 \mathrm{Vrms}$, from $4 \mathrm{Vrms}$ to $10 \mathrm{Vrms}$. Based on the experimental results, the rejection level of the device is as low as $\sim-45 \mathrm{~dB}$ during tuning of the LC. In addition, a wide range of undesired signals is expected to be rejected when the device is implemented in UWB systems. When the device is electrically controlled, some undesired frequency bands, such as WiMAX (3.3-3.6 GHz) and WLAN (5.15-5.35 and $5.725-5.825 \mathrm{GHz}$ ), that could cause signal interference in UWB communications are suppressed to below $-25 \mathrm{~dB}$. 


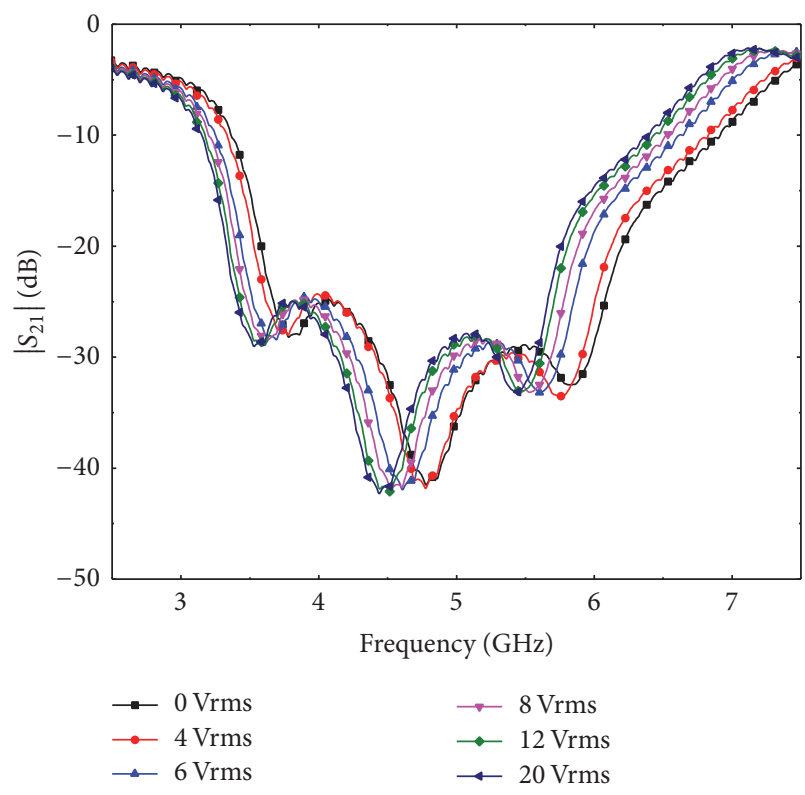

(a)

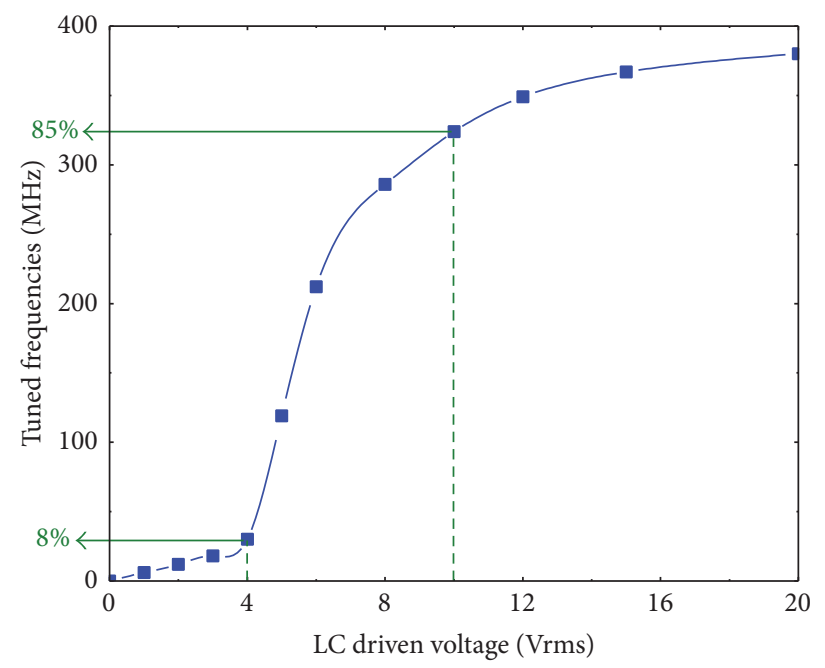

(b)

FIGURE 9: Measured results of (a) transmission $\left|S_{21}\right|$ and (b) tuned frequencies with respect to LC driven voltage.

TABLE 2: Comparison between the simulation and measurement results of the proposed BSF.

\begin{tabular}{lcc}
\hline Parameters & Simulated results & Measured results \\
\hline Frequency (nonbiased/biased) & $4.757 / 4.337 \mathrm{GHz}$ & $4.814 / 4.434 \mathrm{GHz}$ \\
Tuned frequency range/tunability & $0.42 \mathrm{GHz} / 9.7 \%$ & $0.38 \mathrm{GHz} / 8.6 \%$ \\
Transmission $\left|S_{21}\right|$ (nonbiased/biased) & $-53.6 /-58.7 \mathrm{~dB}$ & $-44.2 /-45.8 \mathrm{~dB}$ \\
$3 \mathrm{~dB}$ bandwidth/FBW & $4.73 \mathrm{GHz} / 96.8 \%$ & $4.86 \mathrm{GHz} / 96.2 \%$ \\
$25 \mathrm{~dB}$ bandwidth/FBW & $2.54 \mathrm{GHz} / 52.6 \%$ & $2.51 \mathrm{GHz} / 51.2 \%$ \\
\hline
\end{tabular}

To further verify the design quality of the fabricated device, both the simulation and measurement results are compared in Figure 10. In Figure 10(a), the curves for 0 Vrms and $20 \mathrm{Vrms}$ in the simulation correspond to $\varepsilon^{\prime}=\varepsilon_{\perp}$ and $\varepsilon^{\prime}=\varepsilon_{\|}$of the LC material, respectively. The figure shows that there is a close agreement between the simulation and measurement of the device in terms of the trend of $S$ parameters, though some slight discrepancies remain. One noticeable discrepancy in Figure 10(a) is that there is a small shift between the simulated and measured stopband frequencies when the filter is tuned by a biased voltage of 20 Vrms. This could be due to an error in measurement of the relative permittivity of the LC material. It might be also because a biased voltage of $20 \mathrm{Vrms}$ is not sufficiently high to drive such a thick LC layer $(0.113 \mathrm{~mm})$ to reach the homeotropic orientation state $\left(\varepsilon_{\|}\right)$, though the $S$-parameters of the device are almost unchanged when the biased voltage varies from 15 to $20 \mathrm{Vrms}$. The left plot of Figure 10(a) shows that at 0 Vrms the measured stopband centre frequency closely matches that of the simulated results, which further supports this assumption. Nevertheless, the measured tuning frequency range $(0.38 \mathrm{GHz})$ already reaches $90.5 \%$ of the targeted value $(0.42 \mathrm{GHz})$ at 20 Vrms. Figure $10(\mathrm{~b})$ also shows a good consistency between the measurement and simulation, being the results for the empty device using air as a medium before LC injection. This indicates the accuracy of the model. In addition to the accuracy of the simulation, other potential reasons for deviation between the simulation and measurement include radiation loss due to the lack of metal shielding during measurement, the existence of LC alignment layer, and LC thickness variation resulting from the fabrication process. Table 2 shows a comparison of the performance between the simulation and measurement of the proposed LC based BSF. Table 3 shows a comparison between this work and other LC based tunable BSFs recently reported for C-band and S-band applications. The $3 \mathrm{~dB}$ and $25 \mathrm{~dB}$ bandwidths of this work remain the largest. Though the dielectric anisotropy $\Delta \varepsilon$ of the LC used in this work with $\Delta \varepsilon=0.8$ is not as large as that used in [22] with $\Delta \varepsilon=1.4$, its tunability is still reasonably high.

\section{Conclusions}

Aiming for UWB applications, a compact tunable BSF with an ultra-wide and sharp stopband as well as a large attenuation characteristic has been developed. The proposed 

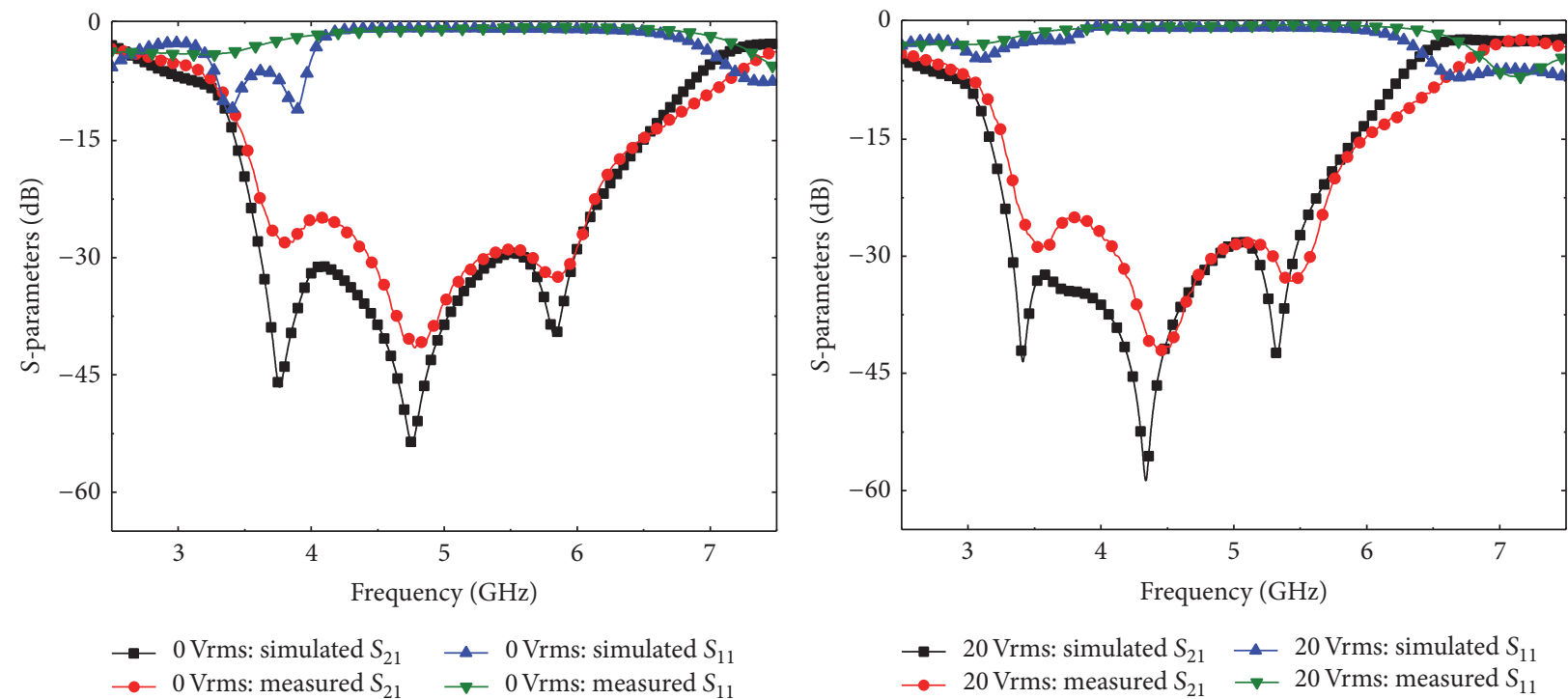

(a)

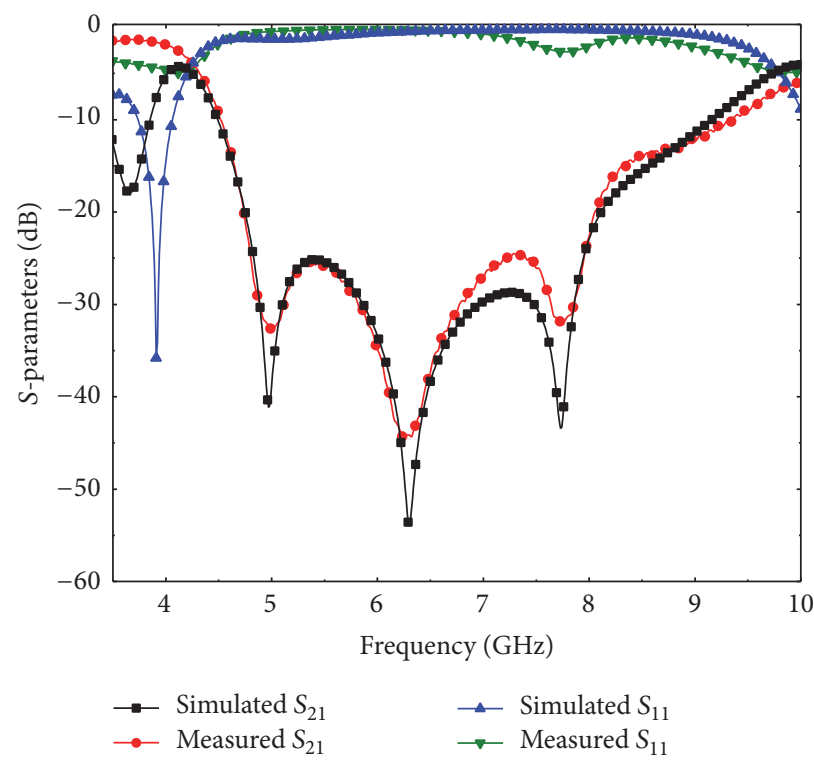

(b)

FIGURE 10: Comparison between the simulation and measurement results of the proposed BSF: (a) LC as the substrate and (b) air as the substrate (before LC injection).

TABLE 3: Comparison with recently reported LC based tunable BSFs.

\begin{tabular}{|c|c|c|c|c|c|}
\hline \multirow{2}{*}{ Parameter } & \multicolumn{5}{|c|}{ Ref. } \\
\hline & {$[16]$} & {$[20]$} & {$[21]$} & {$[22]$} & This work \\
\hline Dielectric anisotropy $\Delta \varepsilon$ & 0.37307 & NA & NA & 1.4 & 0.8 \\
\hline$f_{0}(\mathrm{GHz})$ at $\varepsilon_{r \|}$ & 3.845 & 4.45 & 3.4 & 2.225 & 4.434 \\
\hline Tunable range $(\mathrm{GHz})$ & 0.29 & 0.4 & 0.35 & 0.275 & 0.38 \\
\hline Tunability (\%) & 7.3 & 8.6 & 9.8 & 11.6 & 8.6 \\
\hline $3 \mathrm{~dB}$ bandwidth $(\mathrm{GHz})$ & 3.135 & 1.15 & 1.25 & 1.5 & 4.86 \\
\hline 3 dB FBW (\%) & 81.5 & 25.8 & 36.8 & 67.4 & 96.2 \\
\hline $25 \mathrm{~dB}$ bandwidth $(\mathrm{GHz})$ & $\sim 1$ & 0 & 0 & $\sim 0.7$ & 2.51 \\
\hline Rejection level (dB) & $<-41$ & $<-25$ & $<-20$ & $<-60$ & $<-45$ \\
\hline
\end{tabular}


filter topology uses a wave interference filtering section that consists of three stubs of different lengths, each with an individual stopband of its own central frequency. Equations and guidelines to apply the new wave interference technique to adjust the rejection level and width of its stopband are described. Careful treatment of the main ML and bent stubs was carried out for compactness as well as minimising the device reflection and insertion loss. The measured results of the designed filter show a good agreement with that of the simulation. The centre frequency of the superposed stopband of this filter in measurement can be tuned over a large frequency range between 4.434 and $4.814 \mathrm{GHz}$, nearly $10 \%$ of the central frequency, due to the use of a tunable dielectric medium-LC. The reported BSF possesses other unique features, such as an ultra-wide and sharp stopband, efficient noise attenuation $(\sim-45 \mathrm{~dB})$, and a compact size ideal for being integrated into various monolithic microwave integrated circuits (MMIC). The good performance of the designed BSF makes it potentially useful in UWB and targeted communication systems, including those in extreme and hazardous conditions with special measures such as slow blackening treatments [24] for protections.

\section{Competing Interests}

The authors declare that there is no conflict of interests regarding the publication of this paper.

\section{Acknowledgments}

The authors would like to thank the UK Engineering and Physical Sciences Research Council (EPSRC) for the support through the Platform Grant for Liquid Crystal Photonics (EP/F00897X/1). Longzhu Cai thanks Chinese Scholarship Council and Cambridge Commonwealth, European and International Trust for financial support.

\section{References}

[1] W. J. Feng, W. Q. Che, S. Y. Shi, and Q. Xue, "Compact dual-wideband bandstop filters based on open-coupled lines and transversal signal-interaction concepts," IET Microwaves, Antennas and Propagation, vol. 7, no. 2, pp. 92-97, 2013.

[2] B. Shrestha and N.-Y. Kim, "Microstrip wideband bandstop filter with open stubs for UWB applications," Microwave and Optical Technology Letters, vol. 57, no. 4, pp. 1003-1006, 2015.

[3] A. Svensson, A. Nallanathan, and A. Tewfik, "Ultra-wideband communication systems: technology and applications," Eurasip Journal on Wireless Communications and Networking, vol. 2006, Article ID 16497, 2007.

[4] M. Mokhtaari and J. Bornemann, "Microstrip Ultra-wideband filter with flexible notch characteristics," Wireless Engineering and Technology, vol. 3, no. 1, pp. 12-17, 2012.

[5] J. Wells, "MM-waves in the living room: the future of wireless high definition multimedia," Microwave Journal, vol. 52, no. 8, pp. 72-84, 2009.

[6] B. Allen, A. Brown, K. Schwieger et al., "Ultra wideband: applications, technology and future perspectives," in Proceedings of the International Workshop on Convergent Technologies (IWCT '05), pp. 1-6, Oulu, Finland, June 2005.

[7] T. K. K. Tsang and M. N. El-Gamal, "Ultra-wideband (UWB) communications systems: an overview," in Proceedings of the $3 \mathrm{rd}$ International IEEE Northeast Workshop on Circuits and Systems Conference (NEWCAS '05), pp. 381-386, Québec, Canada, June 2005.

[8] F. Zhu, S. Gao, J. Z. Li, and J. D. Xu, "Planar asymmetrical ultra-wideband antenna with improved multiple band-notched characteristics," Electronics Letters, vol. 48, no. 11, pp. 615-617, 2012.

[9] Z.-P. Li, T. Su, and C.-H. Liang, "Compact wideband microstrip bandpass filter with wide upper stopband based on coupledstub loaded resonator," International Journal of $R F$ and Microwave Computer-Aided Engineering, vol. 25, no. 2, pp. 122128, 2015.

[10] R. K. Mahajan, B. Shrestha, and N.-Y. Kim, "Microstrip symmetrical twin-spiral inductor resonator filter for UWB applications," Arabian Journal for Science and Engineering, vol. 38, no. 9, pp. 2465-2472, 2013.

[11] M. K. Mandal, K. Divyabramham, and V. K. Velidi, "Compact wideband bandstop filter with five transmission zeros," IEEE Microwave and Wireless Components Letters, vol. 22, no. 1, pp. 4-6, 2012.

[12] M. Á. Sanchez-Soriano, G. Torregrosa-Penalva, and E. Bronchalo, "Compact wideband bandstop filter with four transmission zeros," IEEE Microwave and Wireless Components Letters, vol. 20, no. 6, pp. 313-315, 2010.

[13] P. Yaghmaee, O. H. Karabey, B. Bates, C. Fumeaux, and R. Jakoby, "Electrically tuned microwave devices using liquid crystal technology," International Journal of Antennas and Propagation, vol. 2013, Article ID 824214, 9 pages, 2013.

[14] S. Yang, "Simulation of a bandstop filter with two open stubs and asymmetrical double spurlines," International Journal of Advanced Research in Electrical, Electronics and Instrumentation Engineering, vol. 3, no. 9, pp. 11697-11701, 2014.

[15] W.-H. Tu and K. Chang, "Compact microstrip bandstop filter using open stub and spurline," IEEE Microwave and Wireless Components Letters, vol. 15, no. 4, pp. 268-270, 2005.

[16] L. Cai, H. Xu, M. Pivnenko, and D. Chu, "A tunable wideband microstrip bandstop filter based on liquid crystal materials," in Proceedings of the IEEE International Conference on Communication Problem-Solving (ICCP '14), pp. 656-657, December 2014.

[17] L. Cai, H. Xu, J. Li, and D. Chu, "High figure-of-merit compact phase shifters based on liquid crystal material for $1-10 \mathrm{GHz}$ applications," Japanese Journal of Applied Physics, vol. 56, no. 1, Article ID 011701, 2017.

[18] L. Cai, H. Xu, J. Li, and D. Chu, "High FoM liquid crystal based microstrip phase shifter for phased array antennas," in Proceedings of the International Symposium on Antennas and Propagation, Okinawa, Japan, October 2016.

[19] O. H. Karabey, "Liquid crystal material for microwave applications," in Electronic Beam Steering and Polarization Agile Planar Antennas in Liquid Crystal Technology, pp. 9-25, Springer International, Cham, Switzerland, 2014.

[20] V. Urruchi, C. Marcos, J. Torrecilla, J. M. Sánchez-Pena, and K. Garbat, "Note: tunable notch filter based on liquid crystal technology for microwave applications," Review of Scientific Instruments, vol. 84, no. 2, Article ID 026102, 2013.

[21] J. Torrecilla, E. Avila-Navarro, C. Marcos et al., "Microwave tunable notch filter based on liquid crystal using spiral spurline 
technology," Microwave and Optical Technology Letters, vol. 55, no. 10, pp. 2420-2423, 2013.

[22] J. Skulski, A. Szymańska, P. Szczepanski, R. Kisiel, and R. S. Romaniuk, "Liquid crystal tunable microwave band stop filters," in Proceedings of the Electron Technology Conference, p. 89022E, Ryn, Poland, July 2013.

[23] J. D. Taylor, Introduction to Ultra-Wideband Radar Systems, CRC Press, 1994.

[24] F. Xing, B. Zhao, and W. Shi, "Study on tunable fabrication of the ultra-black Ni-P film and its blacking mechanism," Electrochimica Acta, vol. 100, pp. 157-163, 2013. 


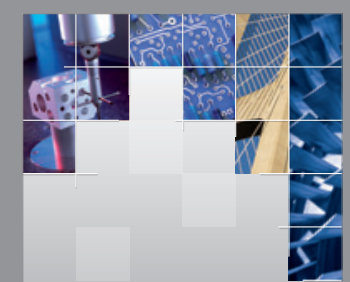

\section{Enfincering}
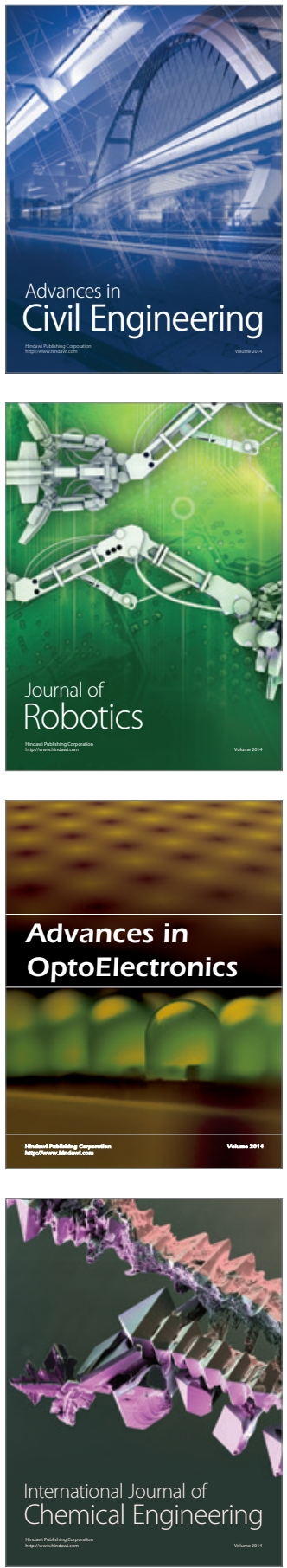

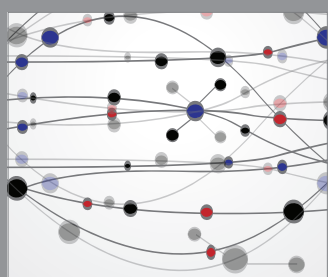

The Scientific World Journal

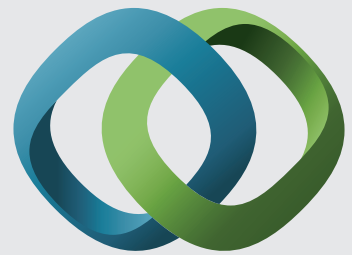

\section{Hindawi}

Submit your manuscripts at

https://www.hindawi.com
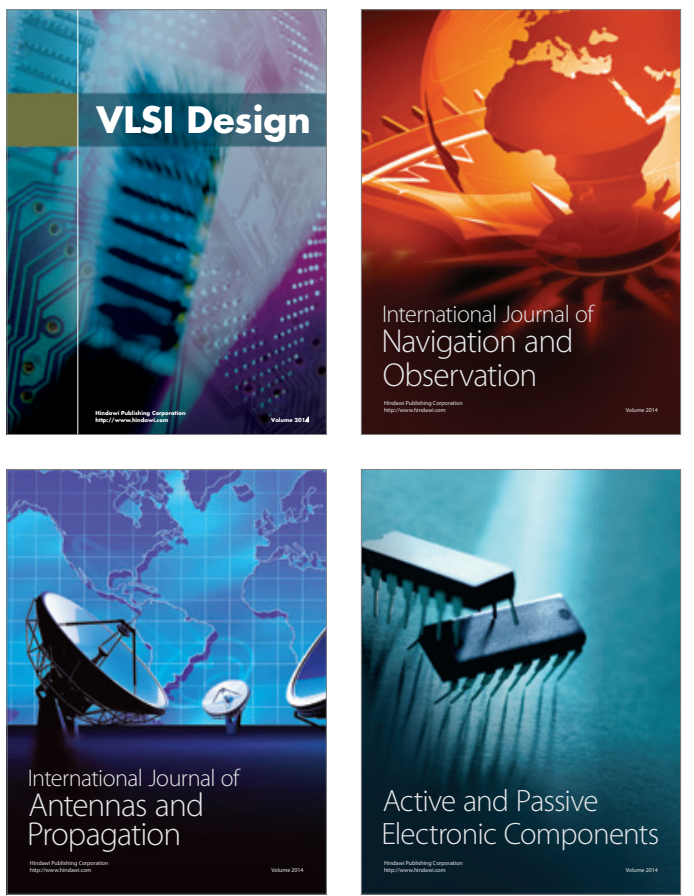
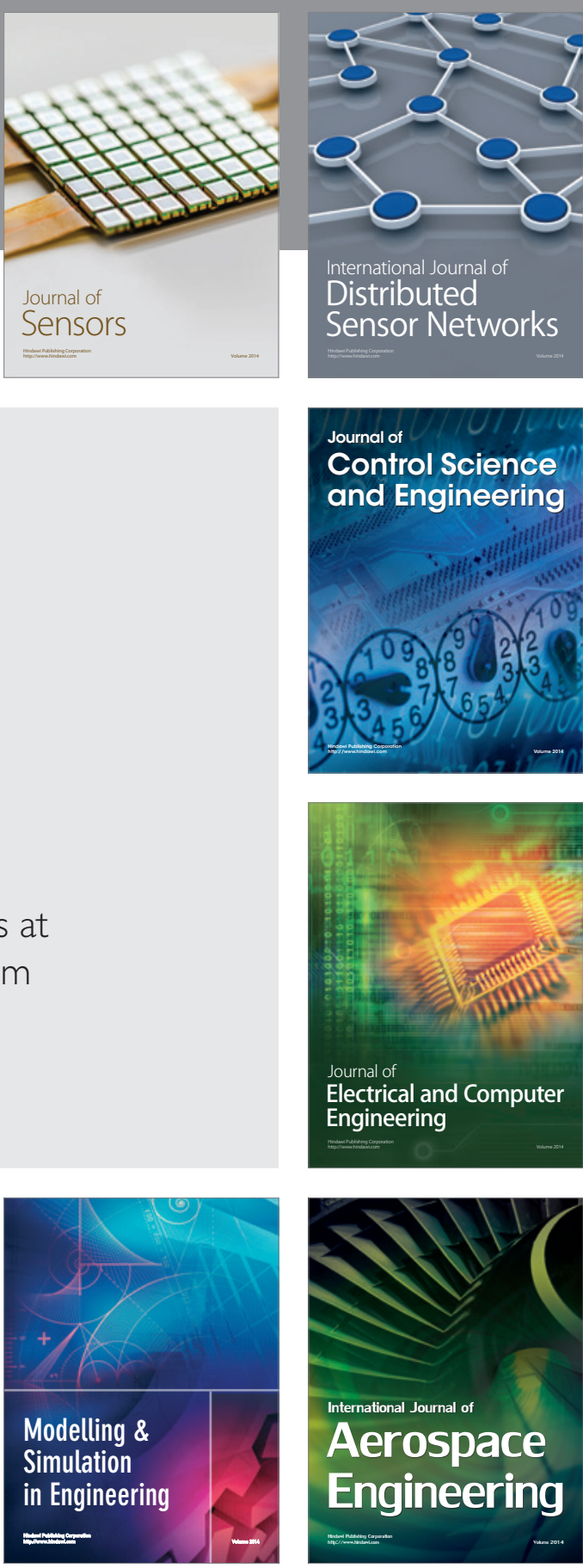

International Journal of

Distributed

Sensor Networks

$-$

Joumal of

Control Science

and Engineering
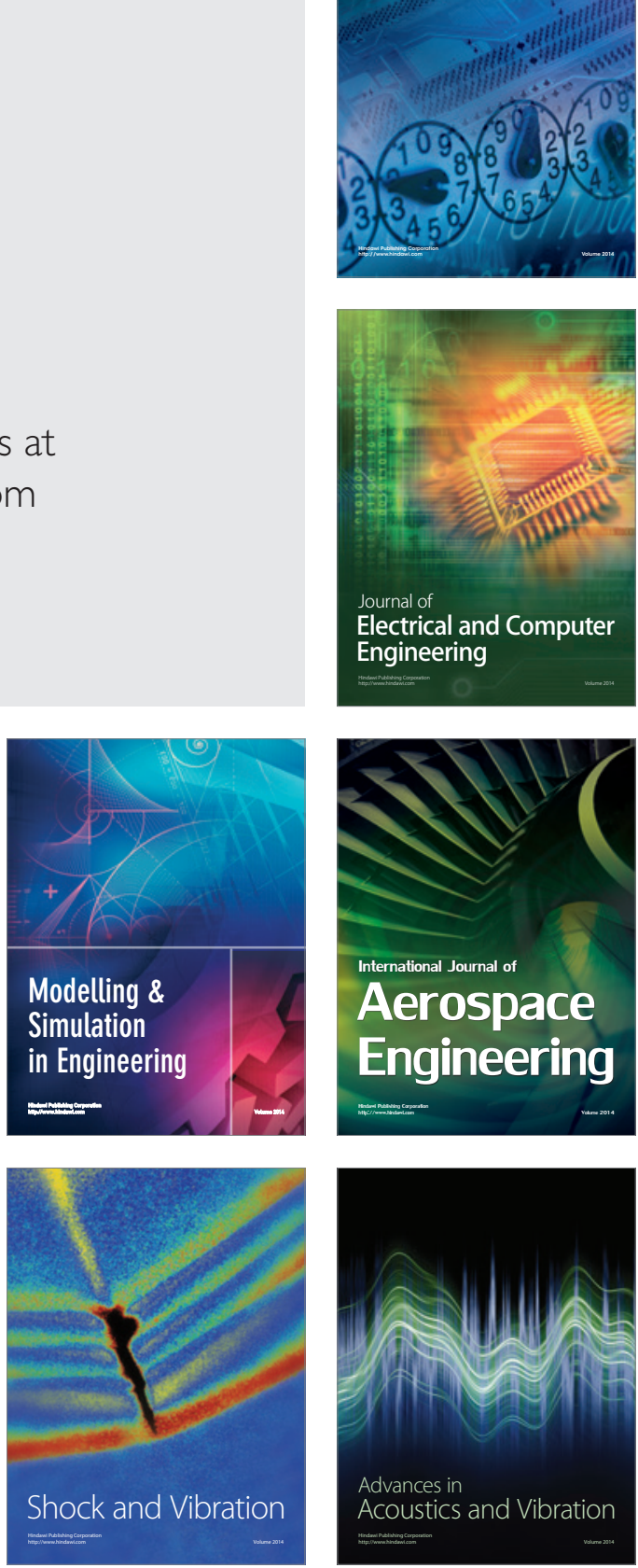